\title{
Mitteilungen des BV ÄLRD
}

Bundesverband

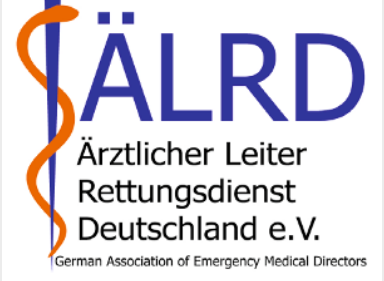

Bundesverband Ärztlicher Leiter Rettungsdienst e.V. Geschäftsstelle

Friedberger Str. 31, 61169 Friedberg

Redaktion:

Prof. Dr. Dr. A. Lechleuthner (Vorsitzender BV ÄLRD, V.i. S.d.P.)

Jutta Schürmann-Lipsch (ÄLRD Märkischer Kreis)

\section{Analgesie-Anwendungen durch Notfallsanitäter: Erste Erfahrungen aus NRW}

Anlässlich eines Landesverbands-Treffens der ÄLRD NRW im Frühjahr 2019 wurden erste Erkenntnisse zum delegierten Einsatz von Analgetika durch Notfallsanitäterinnen und Notfallsanitäter in NRW ausgetauscht. Einzelne Rettungsdienst-Bereiche konnten hierzu aus dem Jahr 2018 bereits Daten auswerten.

Prinzipiell kommen NRW derzeit mindestens drei Konzepte zur Analgesie durch NotSan zur Anwendung:

- Esketamin in Kombination mit Midazolam

- Inhalation von Lachgas-Sauerstoff-Gemisch

- Morphin

In einigen Rettungsdienstbereichen existieren mehrere Konzepte für verschiedene Schmerzbilder parallel.

Aus den Kreisen Mettmann, Borken, Minden-Lübbecke sowie aus den kreisfreien Städten Mönchengladbach und Hamm wurden Erfahrungen mit der Anwendung von Esketamin in Kombination mit Midazolam berichtet. Die Häufigkeit der Anwendung lag zwischen 0,2 \% (35 Anwendungen bei 23.000 RTW-Einsätzen) und 1 \% (115 Anwendungen bei 12.000 RTW-Einsätzen).

Ein Gemisch aus Lachgas und Sauerstoff kommt zur Analgesie derzeit im Kreis Wesel und in der kreisfreien Stadt Münster zur Anwendung. Im Kreis Wesel befindet sich das System in einzelnen Wachen in der Einführung mit bislang nur wenigen Einsätzen (2018: 12 Anwendungen). In Münster wurde das Analgetikum bei 3,3 \% der Einsätze angewendet (780 Anwendungen bei 24.000 RTW-Einsätzen).

Im Kreis Soest, einem ländlich geprägten Flächen-Kreis, wurden 19.000 RTW Einsätze ausgewertet. Es kam in 114 Fällen zur Nutzung von Morphin durch Notfallsanitäter (0,6 \% der Einsätze).

Aus keinem der Rettungsdienst-Bereiche wurden relevante Komplikationen oder Probleme bei der Analgesie durch NotSan berichtet.

Es ist insgesamt festzustellen, dass eine Analgesie durch Notfallsanitäter auf dem Rettungswagen mit 0,2-3 \% aller Einsätze immer noch eine Rarität darstellt, obwohl Daten aus Deutschland mit über $60 \%$ eine sehr hohe Schmerzprävalenz im Rettungsdienst zeigen (Adams HA et al., Anästh Intensivmed 2015; 56: 75-90). Zudem legen aktuelle Daten nahe, dass die Schmerzversorgung im Rettungsdienst häufig noch unzureichend ist. So bleiben $40 \%$ der Patienten mit schmerzhaf- ten Erkrankungen hinsichtlich einer Analgesie unversorgt (Hossfeld B et al.; Notf.med. up2date 2015; 10(03): 269-284).

Ärztliche Leitungen der Rettungsdienste aus den meisten Bereichen des Landes NRW berichten über einen derzeit laufenden Aufbzw. Ausbau von Konzepten zur Analgetika-Anwendung durch Notfallsanitäter. Hierzu sind weitere Publikationen zu erwarten.

\section{Andreas Bohn, Münster}

